\title{
Variedades de arroz locais como alternativas viáveis para uma agricultura mais sustentável
}

\section{Ana Carolina Oliveira Chapeta ${ }^{1}$, Erinaldo Gomes Pereira $^{2}, *$ Alice Firmino Donato ${ }^{3}$, Erika da Costa Fernandes ${ }^{4}$, Leandro Azevedo Santos ${ }^{5}$, Carlos Alberto Bucher $^{6}$, Manlio Silvestre Fernandes ${ }^{5}$ e Cassia Pereira Coelho Bucher ${ }^{2}$}

\begin{abstract}
${ }^{1}$ Universidade Federal Rural do Rio de Janeiro. Instituto de Agronomia. Curso de Pós-Graduação em Engenharia Agrícola e Ambiental. Rodovia BR 465, km 07, s/n. Zona Rural. Seropédica-RJ, Brasil (CEP 23890-000).

${ }^{2}$ Universidade Federal Rural do Rio de Janeiro. Instituto de Agronomia. Curso de Pós-Graduação em Agronomia - Ciência do Solo. Rodovia BR 465, km 07, s/n. Zona Rural. Seropédica-RJ, Brasil (CEP 23890-000). *E-mail: erinaldomn@yahoo.com.br. ${ }^{3}$ Universidade Federal Rural do Rio de Janeiro. Instituto de Agronomia. Curso de Graduação em Agronomia. Rodovia BR 465, km 07, s/n. Zona Rural. Seropédica-RJ, Brasil (CEP 23890-000).

${ }^{4}$ Universidade Federal Rural do Rio de Janeiro. Instituto de Agronomia. Curso de Pós-Graduação em Fitotecnia. Rodovia BR 465, km 07, s/n. Zona Rural. Seropédica-RJ, Brasil (CEP 23890-000).

${ }^{5}$ Universidade Federal Rural do Rio de Janeiro. Instituto de Agronomia. Departamento de Solos. Rodovia BR 465, km 07, s/n. Zona Rural. Seropédica-RJ, Brasil (CEP 23890-000).

6Universidade Federal Rural do Rio de Janeiro. Instituto de Agronomia. Departamento de Fitotecnia. Rodovia BR 465, km 07, s/n. Zona Rural. Seropédica_RJ, Brasil (CEP 23890-000).
\end{abstract}

Resumo. 0 nitrogênio (N) é o macronutriente mais requerido pelas plantas, e a sua deficiência gera grandes perdas para as plantas, tanto na fase vegetativa quanto na fase reprodutiva. Nas plantas, está presente em diversas moléculas e constituintes celulares. No solo, o N está disponível em formas orgânicas, como aminoácidos e peptídeos, e inorgânicas como o nitrato $\left(\mathrm{NO}_{3}^{-}\right)$e amônio $\left(\mathrm{NH}_{4}^{+}\right)$. Devido à alta mobilidade no solo o $\mathrm{NO}_{3}{ }^{-}$é facilmente perdido por processos de lixiviação, volatilização e desnitrificação. 0 objetivo do presente trabalho foi analisar o desenvolvimento radicular e os teores de nitrato de três variedades de arroz, sendo duas variedades locais do Estado do Maranhão, Nordeste do Brasil (Manteiga e Piauí) e uma variedade melhorada (IAC-47), cultivadas em solução nutritiva com baixa concentração de $\mathrm{N}\left(0,2 \mathrm{mM} \mathrm{N}-\mathrm{NO}_{3}^{-}\right)$, durante 30 dias. 0 experimento foi conduzido em câmara de crescimento no Departamento de Solos, da Universidade Federal Rural do Rio de Janeiro (UFRRJ). A variedade Piauí, quando comparada à

Recebido

$23 / 03 / 2020$

Aceito

$15 / 04 / 2020$

Disponível on line $22 / 04 / 2020$

Publicado

$30 / 04 / 2020$

Acesso aberto

ISSN 2359-1412/RBGAS-2020-0036/2020/7/15/16/225 
Manteiga e à IAC-47, apresentou maior desenvolvimento radicular e maior teor de $\mathrm{NO}_{3}-$ nas folhas, enquanto a variedade Manteiga apresentou maior teor de $\mathrm{NO}_{3}^{-}$nas raízes e nas bainhas. Com os resultados obtidos foi possível observar que as variedades de arroz locais do Estado do Maranhão possuem parâmetros mais favoráveis quando cultivadas em ambiente com baixo suprimento de $\mathrm{N}$, e são uma alternativa viável para uma agricultura com baixo uso de insumo.

Palavras-chave: Nitrogênio; Absorção; Assimilação.

Abstract. Local rice varieties as viable alternatives for more sustainable agriculture. Nitrogen $(\mathrm{N})$ is the most required macronutrient by plants, and its deficiency generates large losses to plants, both in the vegetative phase and in the reproductive phase. In plants, it is present in several molecules and cellular constituents. In soil, $\mathrm{N}$ is available in organic forms such as amino acids and peptides, and inorganic forms such as nitrate $\left(\mathrm{NO}_{3}{ }^{-}\right)$and ammonium $\left(\mathrm{NH}_{4}^{+}\right)$. Due to the high mobility in the soil, $\mathrm{NO}_{3}{ }^{-}$is easily lost through leaching, volatilization and denitrification processes. The objective of the present work was to analyze the root development and nitrate content of three rice varieties, two local varieties of rice from Maranhão State, Northeast Brazil (Manteiga and Piauí) and one improved variety (IAC-47), cultivated in nutrient solution with low concentration of $\mathrm{N}(0.2$ $\mathrm{mM} \mathrm{N}-\mathrm{NO}_{3}^{-}$) for 30 days. The experiment was conducted in a growth chamber at the Soil Department of the Federal Rural University of Rio de Janeiro (UFRRJ). The Piauí variety, when compared to Manteiga and to IAC-47, presented higher root development and higher $\mathrm{NO}_{3}$ - content in leaves, while Manteiga variety presented higher $\mathrm{NO}_{3}$ - content in roots and sheaths. With the results obtained it was possible to observe that the local rice varieties of Maranhão State have more favorable parameters when grown in an environment with low supply of $\mathrm{N}$, and are a viable alternative for agriculture with low use of input.

Keywords: Nitrogen; Absorption; Assimilation.

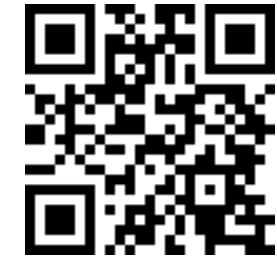

ORCID

(D) 0000-0001-9025-7579

Ana Carolina Oliveira

Chapeta

(D) 0000-0002-9052-091X

Erinaldo Gomes

Pereira

(D) 0000-0001-9773-0074

Alice Firmino Donato

(D) 0000-0003-2960-1881

Erika da Costa

Fernandes

(D) 0000-0002-2595-9432

Leandro Azevedo

Santos

(D) 0000-0002-2746-816X

Carlos Alberto Bucher

(D) 0000-0001-5329-6122

Manlio Silvestre

Fernandes

(D) 0000-0001-5415-1734

Cassia Pereira Coelho Bucher

\section{Introdução}

O nitrogênio $(\mathrm{N})$ é o macronutriente de maior demanda pelas plantas, e a deficiência desse nutriente gera grandes perdas, tanto na fase vegetativa quanto na reprodutiva. Ele está presente em diversas moléculas e constituintes celulares, como nos aminoácidos, proteínas, hormônios vegetais e membranas (Souza e Fernandes, 2018).

No solo o nitrogênio está disponível em formas orgânicas, como aminoácidos e peptídeos, e inorgânicas, como o nitrato $\left(\mathrm{NO}_{3}{ }^{-}\right)$e amônio $\left(\mathrm{NH}_{4}{ }^{+}\right)$.

As plantas diferem na sua preferência por fontes de $\mathrm{N}$, mas o absorvem principalmente sob formas minerais (Williams e Miller, 2001). Porém, os diferentes ambientes de cultivo e as diferentes espécies de plantas exercem influência direta na fonte de $\mathrm{N}$ mineral a ser absorvida em maior quantidade. 
Devido à alta mobilidade no solo, o $\mathrm{NO}_{3}{ }^{-}$é facilmente perdido por processos de lixiviação, volatilização e desnitrificação, sendo esta perda prejudicial sob diversos aspectos. Prejudica as plantas, que ao terem uma menor disponibilidade de $\mathrm{N}$ crescem e produzem menos; prejudica o produtor, o qual deve aumentar a taxa de adubação no intuito de garantir uma produção satisfatória; prejudica o consumidor que arca diretamente com os maiores custos, advindos da maior necessidade de adubação; e prejudica sobretudo o meio ambiente, uma vez que o $\mathrm{NO}_{3}$ - lixiviado ao longo do perfil do solo será depositado em cursos de água, o que, dependendo da quantidade lançada, contribui para a ocorrência de processos de eutrofização de recursos hídricos.

Existem diversas formas de contornar essa problemática, sendo a seleção de genótipos mais eficientes na absorção e assimilação de N, uma das alternativas adotadas por pesquisadores em programas de melhoramento vegetal.

A identificação de variedades naturalmente mais eficientes é um dos pilares dos programas de melhoramento. Estudos feitos com genótipos de arroz demonstraram a existência de diferenças quanto à eficiência no uso de nitrogênio e produção de grãos, indicando uma relação positiva entre os diferentes genótipos e a produtividade (Cancellier et al., 2011; Fageria, 2007).

O Estado do Maranhão, marcado por fluxos intermitentes de nutrientes no solo, devido à alternância de períodos chuvosos e secos, e historicamente um grande produtor de arroz, possui um grande banco de germoplasma, com variedades que guardam consigo diversas adaptações às condições de cultivo presentes naquela região. Características como a maior eficiência no uso de $\mathrm{N}$ e maior desenvolvimento radicular em condições de baixa adubação nitrogenada, vêm sendo identificadas em trabalhos desenvolvidos por pesquisadores no Laboratório de Nutrição Mineral de Plantas (LNMP) (Coelho et al., 2016; Santos et al., 2011).

O objetivo deste trabalho foi realizar uma análise comparativa entre duas variedades locais de arroz do Estado do Maranhão (Manteiga e Piauí) e uma cultivar melhorada (IAC-47), quanto a parâmetros fenotípicos e metabólicos, quando cultivadas à

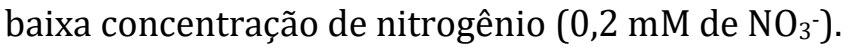

\section{Material e métodos}

0 experimento foi conduzido em câmara de crescimento (luminosidade de aproximadamente $400 \mu$ mols fótons $\mathrm{m}^{-2} \mathrm{~s}^{-1}$; fotoperíodo de $14 \mathrm{~h} / 10 \mathrm{~h}$, umidade de $70 \% \mathrm{e}$ temperatura $28^{\circ} \mathrm{C} / 24^{\circ} \mathrm{C}$ ), no Departamento de Solos, da Universidade Federal Rural do Rio de Janeiro (UFRRJ). Foi utilizado o delineamento experimental inteiramente casualizado, em esquema fatorial $3 \times 1 \times 1$ (variedades $\times$ tratamento $\times$ coleta) com quatro repetições. Para o estudo foram utilizadas as variedades IAC-47 (variedade melhorada), Piauí e Manteiga (variedades locais do Estado do Maranhão).

As sementes foram primeiramente desinfestadas em solução de hipoclorito de sódio a 2,5\%, agitadas por inversão durante $15 \mathrm{~min}$ e depois lavadas com água destilada. Após o processo de desinfestação, as sementes foram levadas para a câmara de crescimento e colocadas para germinar em vasos contendo água destilada. Após 10 dias da germinação, as plântulas foram transferidas para solução nutritiva de Hoagland e Arnon (1950) modificada à meia força iônica $(1 / 2 \mathrm{FI})$, a qual foi renovada a cada três dias.

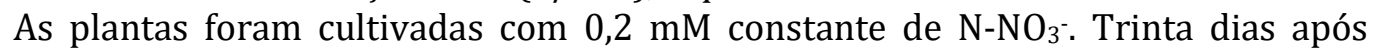
germinação as plantas foram coletadas, seccionadas em raiz (R), bainha (B) e folha (F), e armazenadas para análise de $\mathrm{N}^{-\mathrm{NO}_{3}}$ (Miranda et al., 2001) e morfologia do sistema radicular.

As raízes foram armazenadas em álcool $50 \%$, digitalizadas com auxílio de scanner de mesa (Epson Expression 10000XL). As imagens obtidas após o processo de digitalização foram analisadas com auxílio do programa Winrhizo. 
Os dados foram submetidos à análise de variância e, posteriormente, ao Teste de Tukey à significância de 5\%, utilizando o programa estatístico R (R Core Team, 2019).

\section{Resultados e discussão}

As três variedades em estudo diferiram tanto no desenvolvimento radicular quanto na alocação do $\mathrm{NO}_{3}$.

A variedade Manteiga, comparada à variedade Piauí e IAC-47, apresentou maior teor de $\mathrm{NO}_{3}$ - nas raízes e nas bainhas (Figura 1). No entanto, na parte aérea, esta variedade apresentou menor teor de $\mathrm{NO}_{3}$, demonstrando um acumulo preferencial de NO3- nas raízes e bainhas. Este comportamento pode estar relacionado a um metabolismo mais intenso nas folhas, em detrimento as bainhas e raízes.
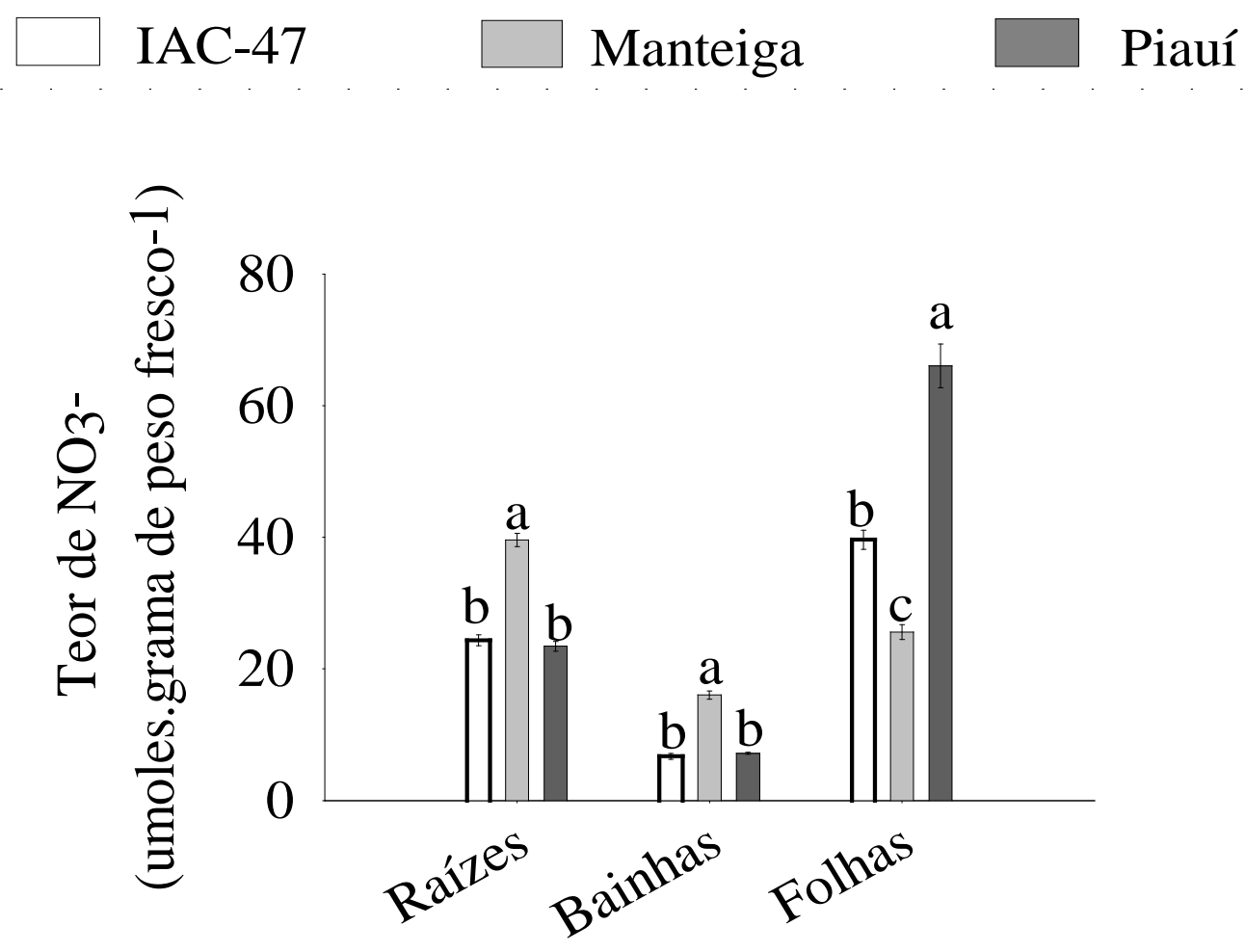

Figura 1. Teores de nitrato nas raízes, bainhas e folhas de plantas de arroz (Oryza sativa L.), variedades IAC-47, Manteiga e Piauí, cultivadas em solução nutritiva com baixa concentração de $\mathrm{N}$ $\left(0,2 \mathrm{mM}\right.$ de $\left.\mathrm{N}-\mathrm{NO}_{3}{ }^{-}\right)$.

A variedade Piauí apresentou o maior teor de $\mathrm{NO}_{3}{ }^{-}$nas folhas, cerca de $40 \%$ superior a variedade melhorada (IAC-47) e 60\% superior a variedade Manteiga. Não diferiu da variedade IAC-47 quanto aos terrores de $\mathrm{NO}_{3}$ - nas raízes e bainhas.

A habilidade que algumas variedades de arroz possuem em estocar parte do $\mathrm{N}$ absorvido durante o estágio inicial de desenvolvimento é considerado um aspecto altamente positivo, pois esta reserva de $\mathrm{N}$ é de suma importância em estágios avançados de desenvolvimento como, por exemplo, durante o período de enchimento de grãos. 
Santos et al. (2009), estudando diferentes variedades de arroz, também observaram o acúmulo de $\mathrm{NO}_{3}{ }^{-}$nas bainhas de variedades de arroz locais, e atribuíram esse comportamento a uma possível estratégia da planta em estocar $\mathrm{N}$ nesse órgão para utilizar em períodos de maior demanda metabólica.

$\mathrm{O}$ acúmulo de altos níveis de $\mathrm{NO}_{3}{ }^{-}$em plantas de arroz, relatado por Fernandes (1974), foi explicado pela existência de um mecanismo de sequestro de $\mathrm{NO}_{3}$, que leva o deslocamento de $\mathrm{NO}_{3}$ - do "pool" metabólico para o "pool" substrato.

Em relação aos parâmetros radiculares, comprimento e número de pontas, pode-se observar diferenças marcantes entre as variedades, sendo que as variedades locais apresentaram parâmetros mais favoráveis ao sistema de cultivo com baixo fornecimento de N (Figura 2A e 2B).

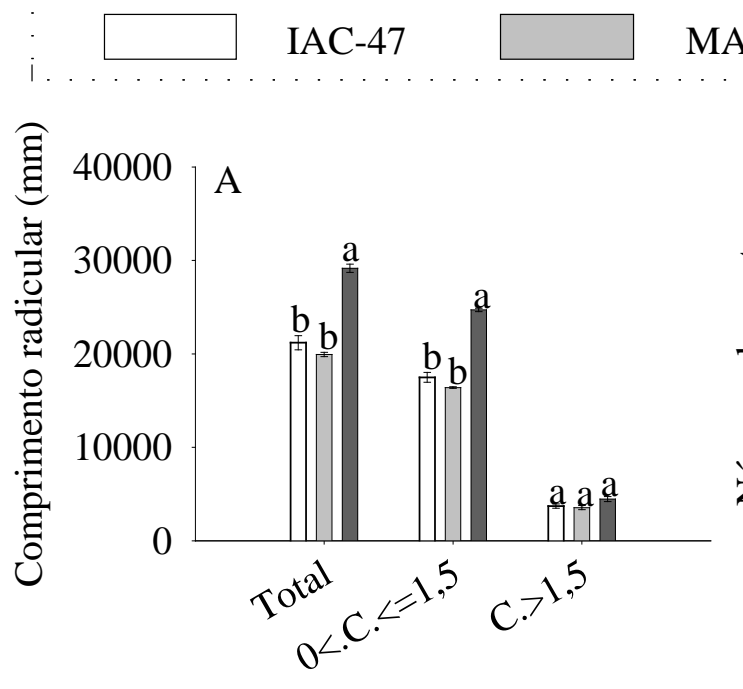

MANTEIGA PIAUÍ

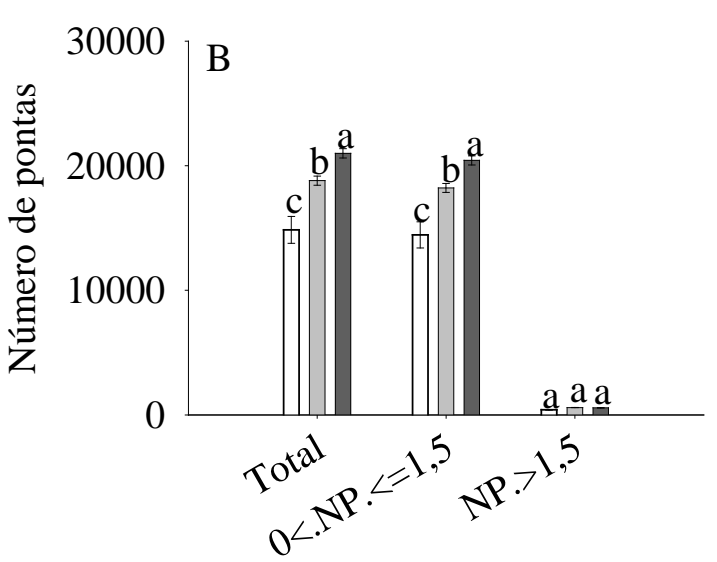

Figura 2. (A) Comprimento radicular e (B) número de pontas: total, 0 a 1,5 mm e maior que 1,5 $\mathrm{mm}$, em três variedades de arroz (IAC-47, Manteiga e Piauí) cultivadas em solução nutritiva com baixa concentração de $\mathrm{N}\left(0,2 \mathrm{mM}\right.$ de $\left.\mathrm{N}-\mathrm{NO}_{3}^{-}\right)$.

Quanto ao comprimento radicular, a variedade Piauí se destacou positivamente, apresentando maior comprimento (total e dentro da classe de raízes mais finas $(0<\mathrm{C}<=1,5))$, o que pode ser interpretado como uma adaptação a esta condição de cultivo (Figura 2A). Não foram encontradas diferenças significativas na classe de raízes grossas $(C>1,5)$. As variedades Manteiga e IAC-47 apresentaram comportamento semelhante para esta variável.

O número de pontas, variável diretamente relacionada a ramificação radicular, foi um parâmetro fortemente contrastante, onde as variedades locais foram muito superiores a variedade melhorada. A variedade Piauí apresentou o maior número de pontas (total e dentro da classe de raízes mais finas $(0<\mathrm{C}<=1,5)$, seguido da variedade Manteiga.

Interessante observar que a superioridade das variedades locais, quanto aos parâmetros radiculares, se concentra na classe de raízes mais finas. Este resultado demonstra uma estratégia dessas variedades em investir em sistemas radiculares mais ramificados e com raízes mais finas, o que proporciona uma maior área de contado do 
sistema radicular com a solução do solo e, certamente, uma vantagem na absorção de nutrientes.

O desenvolvimento radicular superior, apresentado pelas variedades locais, corrobora com os resultados apresentados por Santos et al. (2009).

Como já foi observado por Souza e Fernandes (2018), plantas de arroz submetidas a estresse por deficiência de nitrogênio utilizam reservas presentes na parte aérea e expandem o sistema radicular, no intuito de melhorar a captação do $\mathrm{N}$ presente na solução do solo.

0 maior teor de $\mathrm{NO}_{3}$ apresentado pelas variedades locais, certamente, é consequência dos parâmetros radiculares mais favoráveis apresentados por estas variedades.

A análise de agrupamento obtida pelo dendograma de dissimilaridade fenotípica (Figura 3) e pelo heatmap (Figura 4) demonstra uma clara separação entre as variedades estudadas. As variedades Piauí e IAC-47, por serem as mais contrastantes, formaram grupos muito distintos entre si. Estas análises de agrupamento reforça as diferenças relatadas na arquitetura radicular e no teor de $\mathrm{NO}_{3}$ - (Figuras 1 e 2).

\section{Cluster Dendrogram}

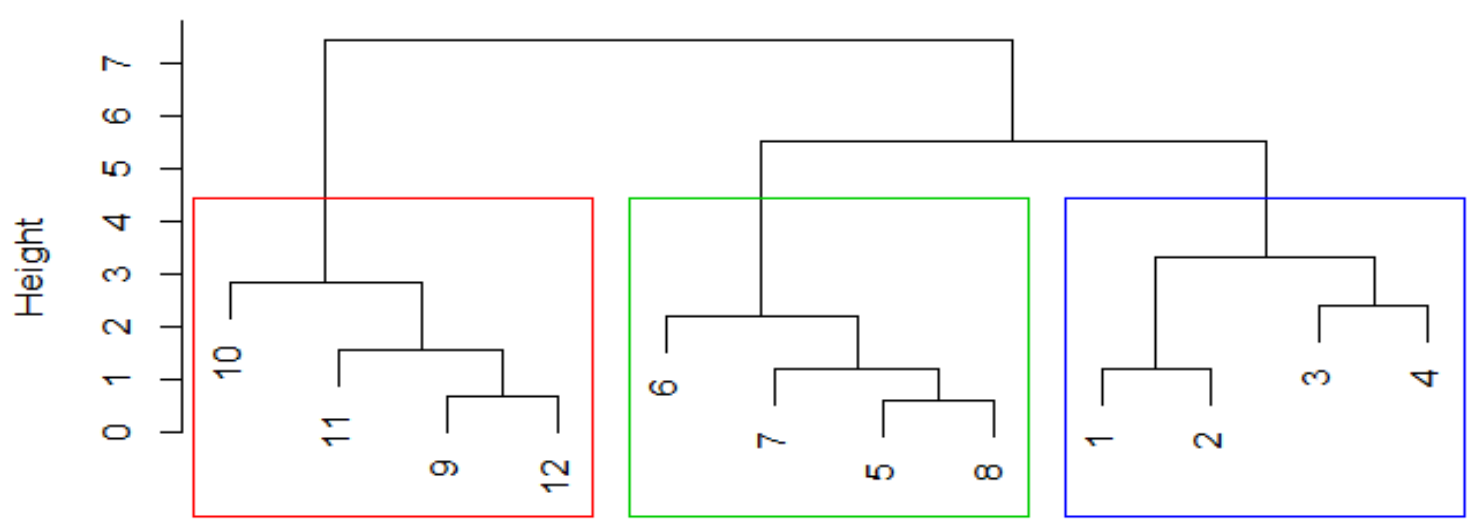

$\operatorname{dist}($ VARIEDADES)

hclust (", "complete")

Figura 3. Dendograma de dissimilaridade fenotípica de três variedades de arroz, $\{1,2,3,4=$ variedade IAC-47\}, $\{5,6,7,8=$ variedade Manteiga $\},\{9,10,11,12=$ variedade Piauí $\}$, cultivadas em solução nutritiva com baixa concentração de $\mathrm{N}\left(0,2 \mathrm{mM}\right.$ de N-NO $\left.3^{-}\right)$.

A variedade Manteiga, por não apresentar características muito contrastantes para os parâmetros analisados, agrupou-se em um nível intermediário.

As características apresentadas pelas variedades locais, após o cultivo em condições de baixo $\mathrm{N}$, se melhor exploradas em programas de melhoramento, pode representar um grande ganho econômico ao produtor, além de um ganho ambiental considerável. Ao produtor por conseguir elevada produtividade com menor uso de insumos. Ao ambiente pela possibilidade de menor deposição de nitrogênio nos corpos 
hídricos, diminuindo a intensidade de processos de eutrofização, tão comuns em regiões agrícolas.

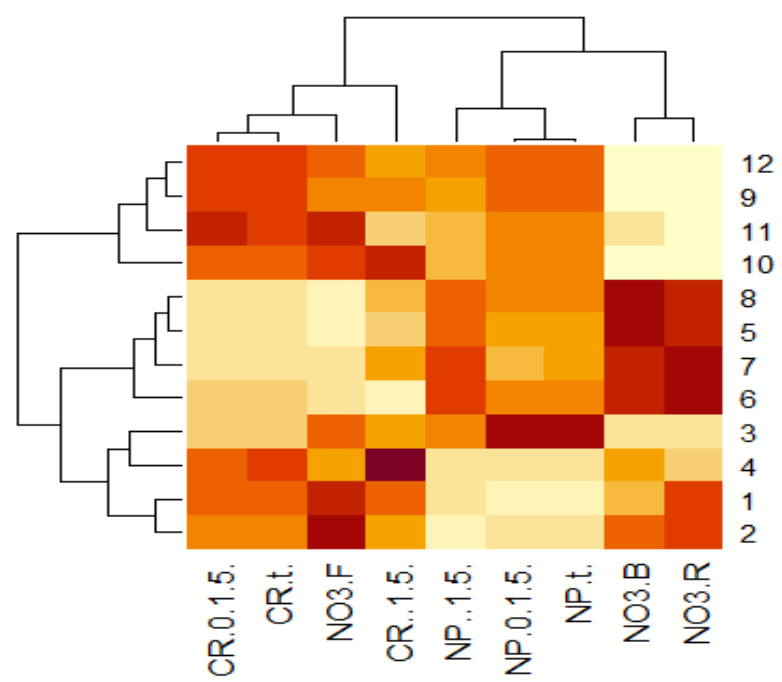

Figura 4. Heatmap dos teores de nitrato nas raízes, bainhas e folhas, comprimento radicular e número de pontas, em plantas de arroz (Oryza sativa L.), variedades IAC-47, Manteiga e Piauí, cultivadas em solução nutritiva com baixa concentração de N (0,2 mM de N-NO${ }^{-}$). CR t; CR 0 - 1.5; CR 1.5; NP t; NP 0 - 1.5; NP 1.5; $\mathrm{NO}_{3}{ }^{-} \mathrm{R}$; $\mathrm{NO}_{3}{ }^{-} \mathrm{B}$; $\mathrm{NO}_{3}{ }^{-} \mathrm{F}=$ Comprimento radicular total, Comprimento radicular (0-1.5 mm), Comprimento radicular $>1.5 \mathrm{~mm}$, Número total de pontas, Número de pontas (0-1.5 mm), Número de pontas > $1.5 \mathrm{~mm}$, teor de nitrato nas raízes, bainhas, folhas, respectivamente.

\section{Conclusões}

As três variedades de arroz diferiram quanto aos parâmetros analisados.

As variedades Manteiga e Piauí apresentaram melhor adaptação a condições de baixo fornecimento de $\mathrm{N}$, demonstrado pelos parâmetros radiculares mais favoráveis.

As variedades locais são altamente promissoras para o uso em agricultura de baixo uso de insumo.

Estudos mais aprofundados são recomendados para o melhor entendimento do comportamento dessas variedades frente a ambientes naturalmente pobres em nitrogênio.

\section{Conflito de interesses}

Os autores declaram não haver conflito de interesses.

\section{Referências}

Cancellier, E. L.; Barros, H. B.; Kischel, E.; Gonzaga, L. A. M.; Brandão, D. R.; Fidelis, R. R. Eficiência agronômica no uso de nitrogênio mineral por cultivares de arroz de terras altas. Revista Brasileira de Ciências Agrárias, v. 6, n. 4, p.650-656, 2011. https://doi.org/10.5039/agraria.v6i4a1420 
Coelho, C. P.; Santos, L. A.; Rangel, R. P.; Sperandio, M. V. L.; Bucher, C. A.; Souza, S. R.; Fernandes, M. S. Rice varieties exhibit different mechanisms for Nitrogen Use Efficiency (NUE). Australian Journal of Crop Science, v. 10, n. 3, p. 342-352, 2016. https://doi.org/10.21475/ajcs.2016.10.03.p7085

Fageria, N. K. Yield physiology of rice. Journal of Plant Nutrition, v. 30, n. 6, p. 843-879, 2007. https://doi.org/10.1080/15226510701374831

Fernandes, M. S. Effects to light and temperature on the nitrogen metabolism of tropical rice. Michigan: Michigan State University, 1974. (Ph.D. Thesis).

Hoagland, D. R.; Arnon, D. I. The water-culture method for growing plants without soil. 2. ed. Berkeley: California Agricultural Experiment Station, 1950. (Circular, 347).

Miranda, K. M.; Espey, M. G.; Wink, D. A. A rapid, simple spectrophotometric method for simultaneous detection of nitrate and nitrite. Nitric Oxide, v. 5, n. 1, p. 62-71, 2001. https://doi.org/10.1006/niox.2000.0319

R Core Team. R: A language and environment for statistical computing. Version 3.5.3. Vienna, Austria: R Foundation for Statistical Computing, 2019. Disponível em: <https://www.r-project.org/>. Acesso em: 23 nov. 2019.

Santos, L. A.; Bucher, C. A.; Souza, S. R.; Fernandes, M. S. Effects of nitrogen stress on proton-pumping and nitrogen metabolism in rice. Journal of Plant Nutrition, v. 32, n. 4, p. 549-564, 2009.

Santos, L. A.; Santos, W. A.; Sperandio, M. V. L.; Bucher, C. A.; Souza, S. R.; Fernandes, M. S. Nitrate uptake kinetics and metabolic parameters in two rice varieties grown in high and low nitrate. Journal of Plant Nutrition, v. 34, n. 7, p. 988-1002, 2011. https://doi.org/10.1080/01904167.2011.555581

Souza, S. R.; Fernandes, M. S. Nitrogênio. In: Fernandes, M. S.; Souza, S. R.; Santos, L. A. (Org.). Nutrição mineral de plantas. Viçosa: Sociedade Brasileira de Ciência do Solo, 2018. v. 2, p. 309-376.

Williams, L. E.; Miller, A. J. Transporters responsible for the uptake and partitioning of nitrogenous solutes. Annual review of Plant Biology, v. 52, n. 1, p. 659-688, 2001. https://doi.org/10.1146/annurev.arplant.52.1.659

Informação da Licença: Este é um artigo Open Access distribuído sob os termos da Licença Creative Commons Attribution, que permite uso irrestrito, distribuição e reprodução em qualquer meio, desde que a obra original seja devidamente citada. 